\title{
The Advanced Light Source Upgrade
}

\author{
D.S. Chemla, B. Feinberg, Z. Hussain, G.F. Krebs, H.A. Padmore, \\ D.S. Robin, A.L. Robinson, and N.V. Smith
}

Lawrence Berkeley National Laboratory,, Berkeley, CA 94720 USA

\begin{abstract}
The ALS, a third-generation synchrotron light source at Berkeley Lab, has been operating for almost a decade and is generating forefront science by exploiting the high brightness of a third-generation source in three areas: (1) high resolving power for spectroscopy; (2) high spatial resolution for microscopy and spectromicroscopy; and (3) high coherence for experiments such as speckle. However, the ALS was one of the first third-generation machines to be designed, and accelerator and insertion-device technology have significantly changed since its conception. As a result, its performance will inevitably be outstripped by newer, more advanced sources. To remain competitive and then set a new standard, the performance of the ALS, in particular its brightness, must be enhanced. Substantial improvements in brightness and current have always been feasible in principle, but they incur the penalty of a much reduced lifetime, which is totally unacceptable to our users. Significant brightness improvements can be realized in the core soft x-ray region by going to top-off operation, where injection would be quasi-continuous and the lifetime objections disappear. In top-off mode with higher average current, a reduced vertical emittance and beta function, and small-gap permanentmagnet or superconducting insertion devices, one to two orders of magnitude improvement in brightness can be had in the soft $\mathrm{x}$-ray range. These improvements also extend the high energy range of the undulator radiation beyond the current limit of $2000 \mathrm{eV}$. Descriptions of the upgrade and the important new science achievable are presented.
\end{abstract}

\section{PUSHING THE SCIENCE LIMITS}

Exploitation of the high brightness of a third-generation synchrotron light source translates into three areas: (1) high resolving power for spectroscopy; (2) high spatial resolution for microscopy and spectromicroscopy; and (3) high coherence for experiments such as speckle. Here we explore the status of each and what would it take to make the next leap. Briefly, the ALS upgrade we propose to go to full-energy injection and higher current and to replace five obsolescent insertion devises with nine state-of-the art insertion devices and four new beamlines to be identified. Moreover, there would be no major disruption to the experimental program of the ALS, since the upgrade could be accomplished in a phased sequence of short (six week) shutdowns.

\section{High Resolving Power Applications.}

A scientific area that has benefited enormously from the availability of high brightness sources is the physics of complex materials studied by photoemission with high energy and high momentum resolution. Improvement in the energy resolution from $50 \mathrm{meV}$ to $10 \mathrm{meV}$ has enabled investigation of low-energy excitations, such as the dispersion "kink" and bilayer splitting in the high $\mathrm{T}_{\mathrm{c}}$ superconductors $[1,2]$. Five photoemission papers have made it to the "ten most cited physics papers". Further improvement in resolution down to the meV range will provide even sharper experimental incisiveness for the understanding of complex materials ranging from strongly correlated electron systems and magnetic materials to systems with reduced dimensionality.

A complementary but more demanding spectroscopy is inelastic x-ray scattering (IXS). Since this is a photonin/photon-out technique, it has the advantage over photoemission of greater penetration and the ability to look at bulk properties and buried interfaces. IXS is the only direct probe of charge-charge correlations that offers momentum resolution. Moreover, unlike in photoemission, it is possible, to apply a magnetic field to the sample. The disadvantage of IXS is its inherently low cross-section, so that the state-of-the-art resolution is $250 \mathrm{meV}$. We have dedicated our last remaining straight section at the ALS to a new beamline for IXS that will reach a resolution of $10 \mathrm{meV}$. This will occur only below a photon energy of $100 \mathrm{eV}$, so that only momentum transfers close to the 
Brillouin-zone center $(\mathrm{q}=0)$ can be investigated. To reach the zone edge it would be necessary to work closer to $1000 \mathrm{eV}$. This can in principle be done but only after the upgrade described below.

\section{High Spatial Resolution Applications}

Understanding fundamental magnetic interactions is at the frontier of solid-state physics but also driven by the technology of thin-film magnetic devices. At the ALS we have pioneered the development of new techniques, one example being the elucidation of the exchange bias in the coupling of the antiferromagnetic (AF) to ferromagnetic (FM) materials that lies at the heart of magnetic spin valves. This problem could only be solved with our new ability to image AF and FM domain structures at high spatial resolution, with interfacial sensitivity, and with elemental and chemical selectivity, by means of photoemission electron microscopy (PEEM). These techniques are revolutionizing our understanding of thin-film magnetism [4-8], and we are hard at work on PEEM3, the next-generation microscope.

Manipulation of spin directly by optical or electrical means leads to the concept of "spintronics"[9]. The fundamental lateral length scale now shrinks to the magnetic exchange length measured in nanometers with a temporal scale set by magnetization precession time of picoseconds. On the horizon are devices such as spin transistors, spin-transfer devices or even spin-based quantum computers. Together with complementary laser probes, time-resolved soft x-ray probes will lead to major advances. Experiments in this domain are brightness limited and are at the edge of viability at the ALS today. They would become practical with the enhancements envisioned for this upgrade.

\section{Coherence Applications}

The high brightness of an optimized third generation source translates directly into high average coherent $\mathrm{x}$-ray flux, roughly the same per second as an x-ray FEL per pulse. While the very high peak coherent flux of an FEL is useful for some experiments, the quasi-dc nature of synchrotron radiation is essential for many others. The diffraction of a coherent $\mathrm{x}$-ray beam from a sample has information at the spatial scale of the wavelength and over a lateral dimension of the coherence width. Two ways to use the information encoded are illustrated below. The upgrade is key in providing much higher coherent flux at the optimum energies).

Zone plate-based x-ray microscopes can now achieve spatial resolutions down to $18 \mathrm{~nm}$ at soft $\mathrm{x}$-ray energies, and with improvements in fabrication, some further progress can be expected. However, for the imaging of threedimensional objects, the thickness of the object places severe restrictions on resolution as the depth of field scales as the square of the resolution. For many applications such as cellular biology, materials engineering, in-situ study of materials in reactive conditions, and nanotechnology, there is a pressing need to find a technique that can look at thick objects at nanometer resolution. It has recently been found that coherent diffraction patterns can be reconstructed back into real space without prior knowledge of phase information. The key is that the transform of a non-periodic object is continuous in Fourier space and can be "oversampled." Electron-density positivity and the sample boundary are then sufficient constraints for convergence on a unique phase set and hence an image [10,11]. We now have the prospect of being able to image thick objects at nanometer resolution in liquids or under reaction conditions. This capability will give us a revolutionary new tool.

The interplay between spin, charge, lattice, and orbital degrees of freedom produces very complex phase diagrams and nanoscale phase behaviors in transition metal oxides. These microphases are thought to play a key role in the properties of high temperature superconductivity in the cuprates and colossal magnetoresistance (CMR) in the manganites. Again, these length scales map directly to soft x-ray probe wavelengths. Soft x-ray coherent x-ray scattering offers important advantages such as high coherent flux, excellent chemical contrast, an adequate scattering wave vector to probe nanoscale features, and access to core levels of interest, such as oxygen and the transition metals. These advantages should enable a much more complete characterization of these materials including their response to external control parameters such as field, temperature and current as well as their related dynamical properties.

\section{TOP OFF AND ADVANCED UNDULATORS FOR INCREASED BRIGHTNESS}

Machine performance of the ALS is lifetime limited. Substantial improvements in brightness and current have always been quite feasible, but they incur the penalty of a much reduced lifetime, an option totally unacceptable to 
our users. In top-off mode, injection would be quasi-continuous, and so the lifetime impediment disappears. At present the ALS is operated in a mode where beam is injected three times daily to $400 \mathrm{~mA}$. In the eight hours between fills, the beam decays to $200 \mathrm{~mA}$ with a time-averaged current of about $250 \mathrm{~mA}$. With an upgrade of the RF system, it is possible to increase the current in the machine to $750 \mathrm{~mA}$. In terms of raw flux, top-off would therefore increase our capacity to the equivalent of three ALSs, which must surely rank as a dramatic example of cost effectiveness. More important than capacity, however, is enhanced capability. The proposed upgrades will enable the newer and more revolutionary experiments briefly outlined above.

For the ALS, the "workhorse" is a $4.45 \mathrm{~m}$ long, $50-\mathrm{mm}$-period linearly polarizing undulator. The ALS has five lof these insertion devices that were designed and installed about a decade ago. In the meantime, undulator technology has undergone significant advances. The currently favored design is the 2-meter elliptically polarizing undulator (EPU). Of these, we have two installed, one under construction, and one proposed. However, insertion-device technology continues to advance, with small-gap, short-period, in-vacuum undulators and superconducting undulators emerging as the insertion devices of choice. The old workhorse device requires one storage ring straight section. In addition to going to top-off operation with higher average current, significant brightness improvements can be realized in the core soft $\mathrm{x}$-ray region by reducing the vertical emittance and beta function and installing small gap, permanent-magnet or superconducting insertion devices. In Figure 1 and Table 2, we compare the brightness of the workhorse using the present storage ring parameters with three other devices using the upgraded ring parameters (Table 1). The new devices all have a magnetic gap of $5 \mathrm{~mm}$. The first two are $28-\mathrm{mm}$-period devices of lengths $4.45 \mathrm{~m}$ (full length) and $2.0 \mathrm{~m}$ (half length) respectively. These two devices would be possible with hybrid permanent magnets $[12,13]$. The third is a 14-mm-period, 1.5-meter-long (half length) superconducting device. The parameters of this device are similar to that being constructed by ACCEL [14].
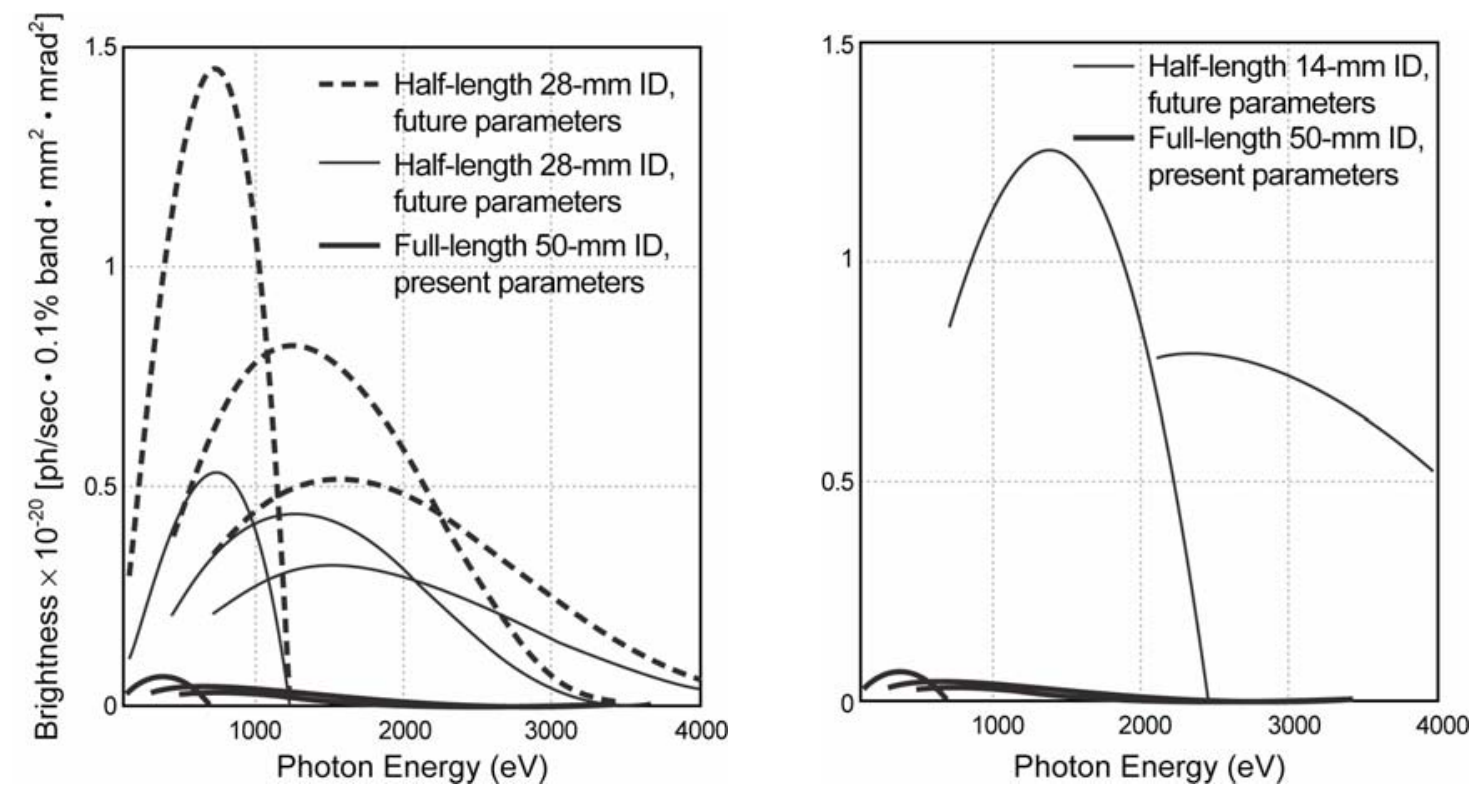

FIGURE 1. Brightness comparison of new ALS insertion devices to current insertion devices

We see that there is one to two orders of magnitude improvement in brightness that can be had in the soft x-ray range. In reducing the vertical emittance after the upgrade, we also reduce the wavelength at which the undulators become diffraction limited to $0.57 \mathrm{~nm}$, i.e., the ALS will be diffraction limited in the vertical direction throughout the whole of the soft x-ray energy range. It should also be noted that the proposed upgrades extend the high-energy range of the undulator radiation, which is currently limited at $2000 \mathrm{eV}$.

TABLE 1. Comparison of Present And Future Storage Ring Parameters

\begin{tabular}{lccc}
\hline Photon Energy & Present & Future & Improvement \\
\hline Time Average Current (mA) & 250 & 750 & 3 \\
Veritcal Emittance (nm•rad) & 0.15 & 0.01 & 15 \\
Vertical Beta Function (m) & 3.6 & 2.25 & 1.5 \\
Vertical Magnetic Gap (mm) & 14 & 5 & 3 \\
\hline
\end{tabular}


TABLE 2. Comparison of Present And Future Brightness (photons $/ \mathrm{sec} / \mathrm{mm}^{2} / \mathrm{mrad}^{2} / 0.01 \%$ BW)

\begin{tabular}{lccc}
\hline Photon Energy & Present Brightness & $\begin{array}{c}\text { Future Brightness } \\
\text { (ID length/gap) }\end{array}$ & Improvement \\
\hline $500 \mathrm{eV}$ & $5.7 \times 10^{18}$ & $1.2 \times 10^{20}($ w/long $28 \mathrm{~mm})$ & 20 \\
& & $4.5 \times 10^{19}($ w/short $28 \mathrm{~mm})$ & 8 \\
$1000 \mathrm{eV}$ & $3.5 \times 10^{18}$ & $1.1 \times 10^{20}($ w/short $14 \mathrm{~mm})$ & 32 \\
$2000 \mathrm{eV}$ & $6.1 \times 10^{17}$ & $8.6 \times 10^{19}($ w/short $14 \mathrm{~mm})$ & 140 \\
$4000 \mathrm{eV}$ & not accessible & $8.1 \times 10^{19}($ w/short $14 \mathrm{~mm})$ & $\infty$ \\
\hline
\end{tabular}

One important aspect of the upgrade is to take some full-length, linearly polarized undulators and replace them with chicaned straights containing two shorter, more advanced devices. With the increased flux and brightness available, these devices will perform far better than the current devices, but at the same time allow simultaneous operation of two fully optimized, independent, application-specific beamlines. The ALS, unlike the larger x-ray machines, has a severely limited number of straight sections. This has meant that several world-class programs that would normally command one or more insertion devices, have multiple end stations sharing a single beamline. One example is the combination of the condensed-matter physics and atomic and molecular physics programs on Beamline 10.0.1, each of which is world leading and would merit a beamline of its own. It would be preferable to design optical systems fully optimized to the type of science on each beamline. In sum, we intend to replace five workhorse insertion devices with nine newer more advanced insertion devices plus four new beamlines that are responsive to the scientific drivers such as those discussed above. We propose an orderly upgrade in which obsolescent insertion devices are progressively replaced by state-of-the-art insertion devices. All upgrades envisioned can be executed in a phased sequence of short (six weeks) shutdowns.

\section{ACKNOWLEDGMENTS}

The authors wish to acknowledge the many members of the ALS staff who contributed to the formulation of these upgrade plans. The list includes but is not limited to W.C. Barry, S. Kwiatkowski, S. Marks, S. Prestemon, F. Sannibale, R. Schlueter, C. Steier, and G. Stover.

\section{REFERENCES}

1. Lanzara, A., et al., "Evidence for ubiquitous strong electron-phonon coupling in high-temperature superconductors," Nature 412, 510-514 (2001).

2. Gromko, A.D., et al, "Mass-renormalized electronic excitations at $(\pi, 0)$ in the superconducting state of $\mathrm{Bi}_{2} \mathrm{Sr}_{2} \mathrm{CaCu}_{2} \mathrm{O}_{8+\mathrm{x}}$ " Cond-mat, 0202329 (2002).

3. Workshop on "Soft X-Ray Science in the Next Millenium: The Future of Photon-In/Photon-Out Experiments," (Pikeville, Tennessee, March 15 - 18, 2000), prepared by Oak Ridge National Laboratory for the U.S. Department of Energy.

4. Scholl, A., et al., "Observation of antiferromagnetic domains in epitaxial thin films," Science 287, 1014-1016 (2000).

5. Stohr, J., et al., "Images of the antiferromagnetic structure of a $\mathrm{NiO}(100)$ surface by means of x-ray magnetic linear dichroism spectromicroscopy," Phys. Rev. Lett. 83, 1862-1865 (1999).

6. Nolting, F., et al., "Direct observation of the alignment of ferromagnetic spins by antiferromagnetic spins," Nature 405 (6788), 767-769 (2000).

7. Ohldag, et al., "Spectroscopic identification and direct imaging of interfacial magnetic spins," Phys. Rev. Lett. 87, 247201/1-4 (2001).

8. Ohldag, H., et al., "Spin reorientation at the antiferromagnetic $\mathrm{NiO}(001)$ surface in response to an adjacent ferromagnet," Phys. Rev. Lett. 86 (13), 2878-2881 (2001), and references therein to ALS magnetic microscopy.

9. Wolf, S.A., et al., " Spintronics: A spin-based electronics vision for the future ," Science 294, 1488-1495 (2001).

10. Miao, J. et al., "High-resolution 3D x-ray diffraction microscopy," Phys. Rev. Lett. 89 , 088303/1-4 (2002).

11. He, H. et al. "Inversion of x-ray diffuse scattering to images using prepared objects," Phys. Rev. B 67, 174114/1-4 (2003).

12. Chavanne, J., P. Elleaume, and P. Van Vaerenbergh, "Recent developments of insertion devices at the ESRF," in Proceedings of the 1999 Particle Accelerator Conference, New York, 1999.

13. Hara, T., T. Tanaka, T. Tanabe, X.M. Marechal, S. Okada, and H. Kitamura, "In-vacuum undulators of SPring-8," J. Synchrotron Rad. 5, 403-405 (1998).

14. Moser, H., and R. Rossmanith, "Magnetic field of superconductive in-vacuo undulators in comparison with permanent magnet undulators," Nucl. Instr. and Meth. A 490, 403-408 (2002). 